\title{
Implementation of Hybrid OFDM-FSO Transmission System
}

\author{
Vishal Sharma \\ SBS STC, Ferozepur \\ Punjab, India
}

\author{
Sushank Chaudhary \\ SBS STC, Ferozepur \\ Punjab, India
}

\begin{abstract}
A novel design based on integration of OFDM and FSO system is reported in this work to prolong the FSO link at high transmission rate under clear weather conditions and evaluates the performance of such hybrid systems using ODSB, OSSB and OTSB schemes. Simulations indicate that the hybrid OFDM-FSO promises considerably improved free space link with acceptable SNR and BER compared to traditional FSO systems.
\end{abstract}

\section{General Terms}

Free Space Optics (FSO) Transmission System

\section{Keywords}

Orthogonal Frequency Division Multiplexing (OFDM), Optical Double Side Band (ODSB), Optical Single Side Band (OSSB), Optical Tandem Side Band (OTSB).

\section{INTRODUCTION}

Free-space Optics (FSO) has the combined features of most dominated telecommunication technologies i.e. Wireless and Fiber Optics. Many of the aspects of FSO are related to fiber optics with an important difference of transmission medium which is air/free space rather than the glass of the fiber-cable. The remarkable growth of internet and other wireless networks had a huge influence in the modern era of telecommunication. Being in the era of ever-present connectivity, the requirement of high speed networks had put the pressure on wireless communication networks to increase both the transmission capacity and the coverage area. The recent trends for ultramodern broadband, interactive and multimedia services over wireless media in both mobile and fixed cellular networks are to reduce cell size to accommodate more users together with to operate in the millimeter wave (mm-wave) frequency bands to avoid spectral congestion in the lower frequency bands [1]. High capacity, low power consumption, light weight, small sizes, high data rates and low costs for satellite cross-links are other promises of FSO technology. It is a significant building block for wide area space networks, supporting mobile users, high speed data services for small satellite terminals and serving as a backbone network for high speed trunking [2]. However, FSO is exceptionally expensive preventing it from replacing the current wireless networks. Improvement of physical-link communication efficiency by an order of magnitude using photon-counting receivers for vacuum channels and by reducing the system complexity, weight, and power for space systems helps in reducing the cost. By incorporating of coherent systems in links and in multiple access applications where coherent processing can reduce the level of interference, a significant reduction in system-cost can be realized [3]. FSO technology is implemented using a laser device which can be mounted on rooftops, corners of buildings or even inside offices. FSO devices look like security video cameras and basically consist of an optical transceiver with a laser transmitter and receiver to provide full duplex capability. FSO unit using a high-power optical source also has a lens that transmits light through the atmosphere to other lens receiving information. If the transmitter source does not produce a sufficiently parallel beam to travel the required distance, the collimation can be done with lens. The receiving lens further connects to a high-sensitivity receiver via optical fiber. The architecture of FSO system can be point to point, mesh or point to multipoint. Low-power infrared beams, which does not harm the eyes, is the other mean by which FSO systems can transmit data through the air between transceivers, or link heads over distances of several hundred meters to a few kilometers, depending upon atmospheric conditions [4]. No doubt, FSO systems can be a good way out for some broadband networks requirements due to many advantages but there are some restrictions that limit its performance. So, it is important to take several FSO system parameters into consideration such as internal- and externalparameters. Most significant external-parameters are rain, dust, snow, fog, or smog that deteriorate the transmission path and shut down the network. These environmental changes are inevitable and must be considered while designing a FSO system. The internal parameters are related to optical power, transmission bandwidth, divergence angle, optical loss on transmitter side, BER, lens diameter and receiver field-ofview (FOV) [5-6].

In case of wireless communication system, a significant effort is done to reduce the multipath fading by introducing OFDM modulation. The OFDM technique distributes the data over a large number of carriers that are spaced apart at precise frequencies with overlapping bands. The use of FFT for modulation provides orthogonality to the subcarriers, which prevents the demodulators from seeing frequencies other than their own [7]. Hence, by incorporating OFDM along with the optical fiber, such OFDM-RoF systems can be used for both short distance as well as long-haul transmission at very high data rates [8]. Based on OFDM technology and optical communication, Optical OFDM (OOFDM) system can construct high-speed, large-capacity and low-cost optical transmission network [9] and its channel capacity is highly scalable, allowing smooth upgraded or transition from existing networks. Combined with RoF technology, OOFDM system can not only reduce multipath fading of wireless signals, but also improve signal quality. Moreover, the system can achieve seamless coverage, increase channel capacity and transmission rate [10]. Demonstrations of integrated OFDMRoF systems have been reported in the previous research work [7-17], but the integration of OFDM and FSO systems has not demonstrated and investigated extensively. In this paper, a demonstration of hybrid OFDM-FSO transmission system, first time, using OPTISYSTEM ${ }^{\mathrm{TM}}$ simulator to establish an FSO link estranged by a distance up to $10 \mathrm{Km}$ at 
data rate of $10 \mathrm{Gbps}$. Further, a comparison is presented for the performance of such systems incorporating ODSB, OTSB and OSSB techniques with-and without- a Bessel filter connected after MZM modulator.

\section{MODEL DESCRIPTION \& RESULTS}

The FSO system consists of three main communication parts which are transmitter, propagation channel and receiver. The FSO system is not much different from fiber optic communication where the difference relies in the propagation medium. In the OPTISYSTEM ${ }^{\mathrm{TM}}$ software, the FSO link is modeled between an optical transmitter and optical receiver with $15 \mathrm{~cm}$ optical antenna at each end. The attenuation of free space between two connecting FSO nodes is considered as $0.11 \mathrm{~dB} / \mathrm{Km}$ under clear weather conditions. The transmitter and receiver gains are $0 \mathrm{~dB}$ by assuming both the antennas ideal. Also, scintillation and mispointing losses are not considered in this simulative work. At transmitting end, a 10Gbps data is generated by using a 4 QAM sequence generator with 2 bit per symbol. This 10Gbps QAM data signals are then OFDM modulated by means of OFDM modulator using 512 subcarriers and FFT size of 1024 to generate 10Gbps-OFDM analog data signals which, further, QAM modulated at $7.5 \mathrm{GHz}$ modulator frequency. This $10 \mathrm{Gbps}$ OFDM treated analog data signals are mixed with RF signal of $17.5 \mathrm{GHz}$ and then transmitted over FSO link by means of different modulation schemes such as ODSB, OTSB, and OSSB using MZM modulator and a phase shifter. A CW laser of $10 \mathrm{MHz}$ with power of $0 \mathrm{dBm}$ at $1550 \mathrm{~nm}$ is used in this simulation. At the base station, a PIN photo detector and a low pass filter is used to recover 10GbpsOFDM data successfully.

This work discussed the results obtained from our proposed simulative setup consisting of 10Gbps-OFDM analog data signals transmitted via FSO link under clear weather conditions. The results are investigated using different modulation schemes such as OSSB, OTSB and ODSB. It is observed that an improvement of $1.5 \mathrm{~dB}$ of SNR is achieved in case of OFDM-OTSB system after transmitting 10Gbps data signals up to an FSO link of $1 \mathrm{~km}$ on comparing with OFDMOSSB system as shown in Fig 3(a). But, beyond 30Km, OFDM-OSSB system behaves better than the OFDM-OTSB system and shows an achievement of $0.42 \mathrm{~dB}$ improvement in SNR after an FSO link of $10 \mathrm{Km}$. Consequently, the total received power at photo detector in case of former transmission system is increased by a factor of $2 \mathrm{~dB}$ than that of the latter one as shown in Fig 3 (b, d) up to $1.5 \mathrm{~km}$ with same acceptable BER of $10^{-6}$ in both the cases under clear weather conditions.

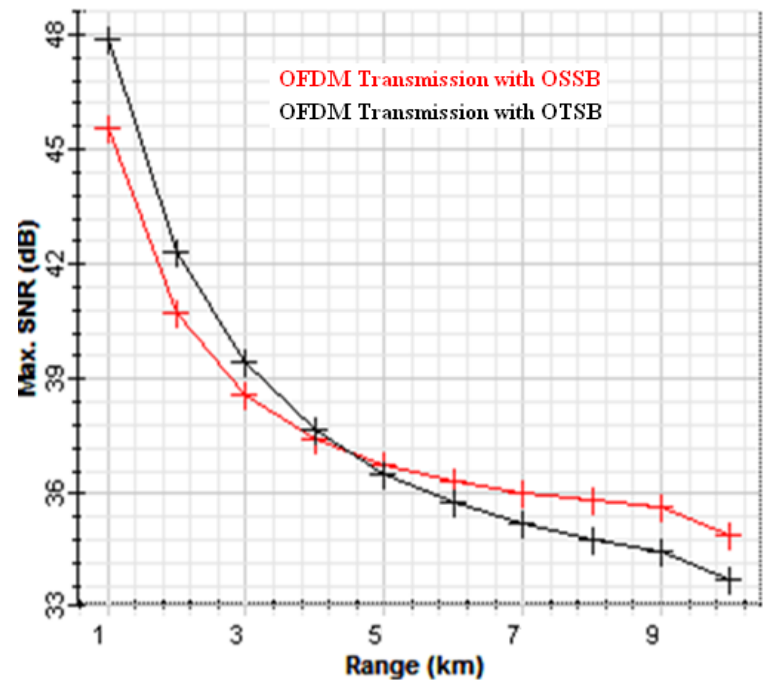

(a)

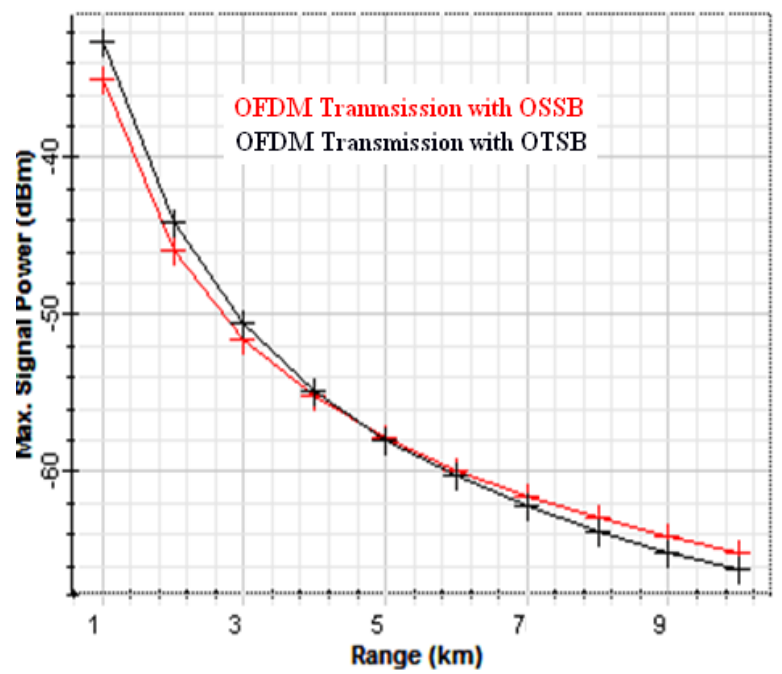

(b)

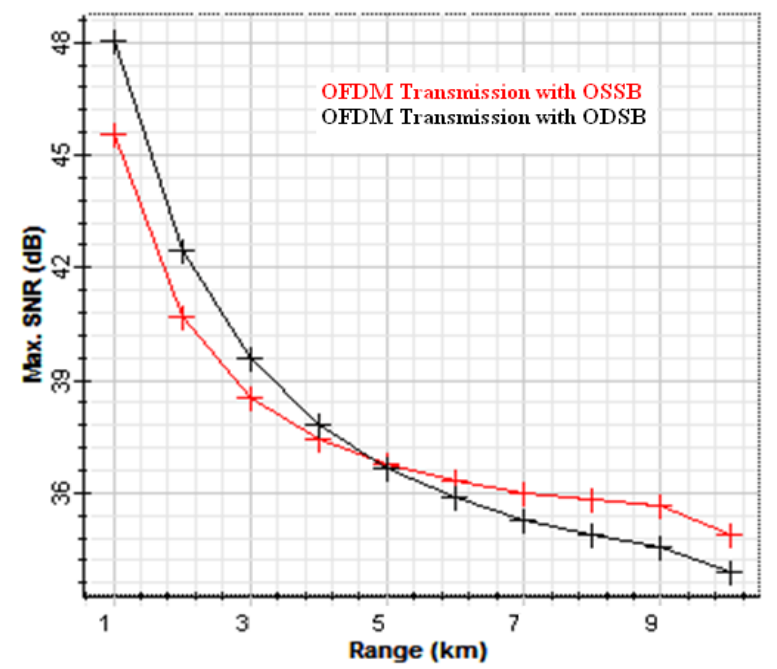

(c) 


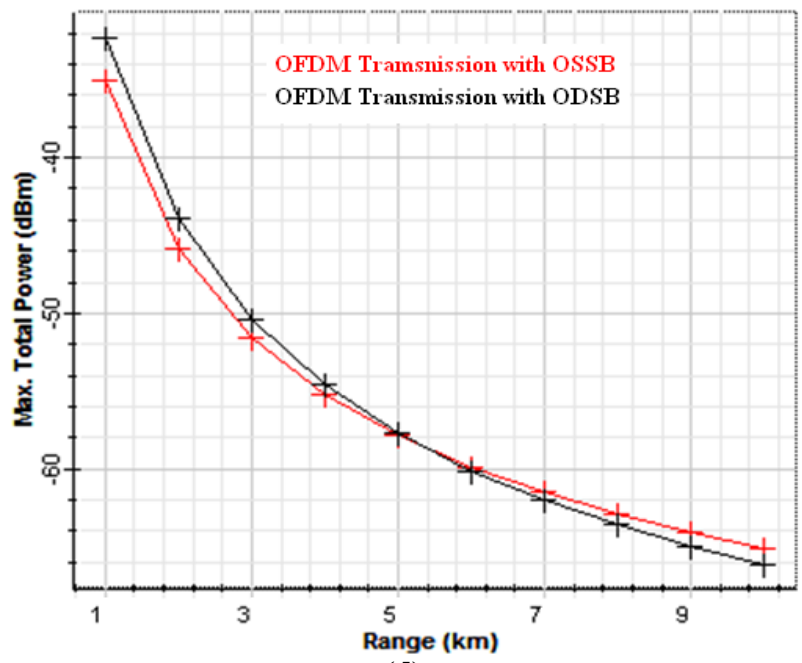

(d)

Fig 1: Evaluation of SNR and Power vs Range of OTSB and OSSB modulation schemes

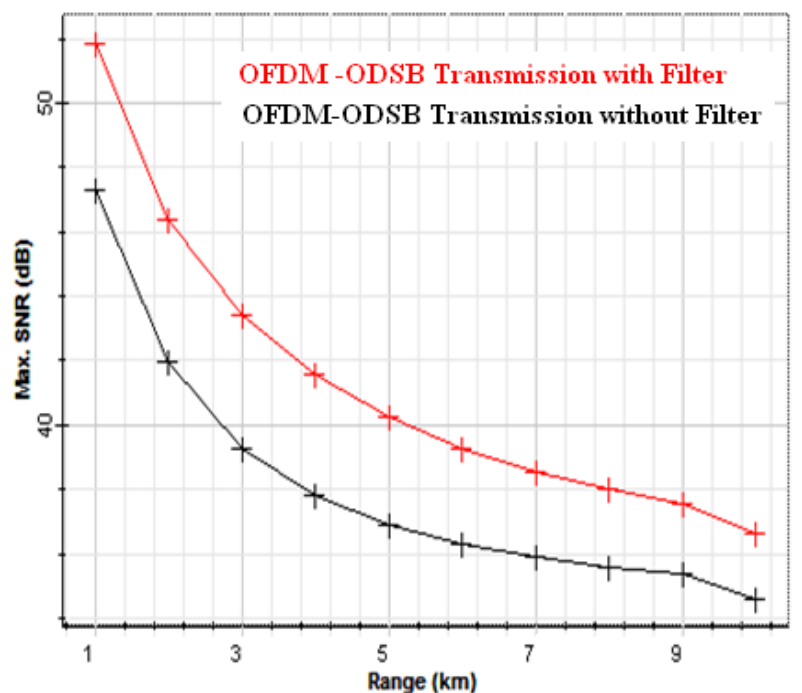

(a)

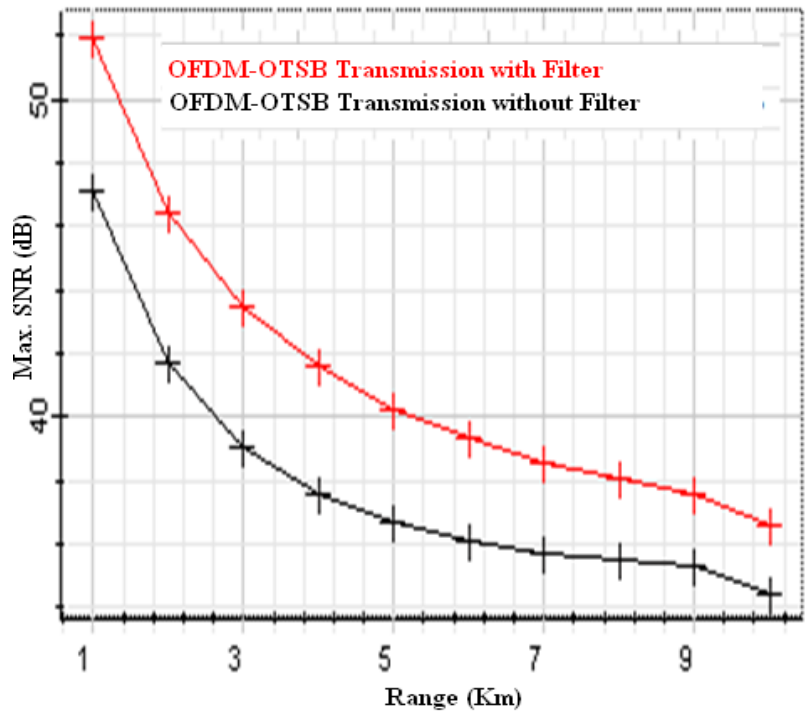

(b)

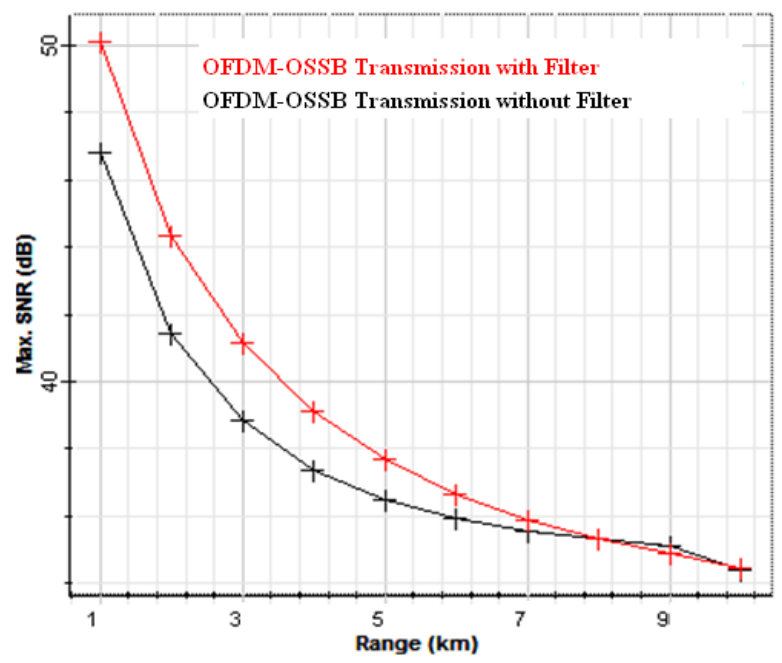

(c)

Fig 2: Evaluation of SNR vs FSO link with (a) ODSB, (b) OTSB, and (c) OSSB modulation schemes with Filter.



(a)

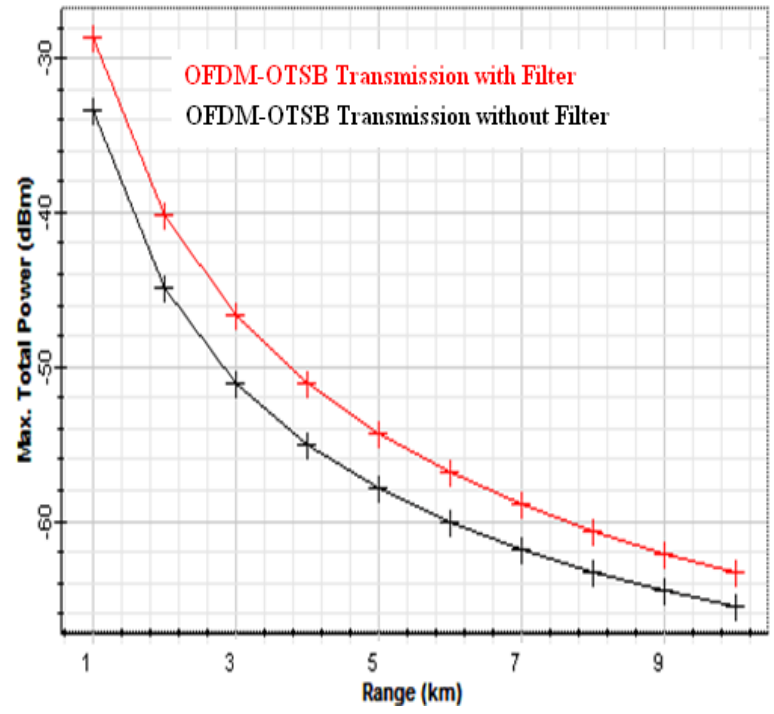

(b) 


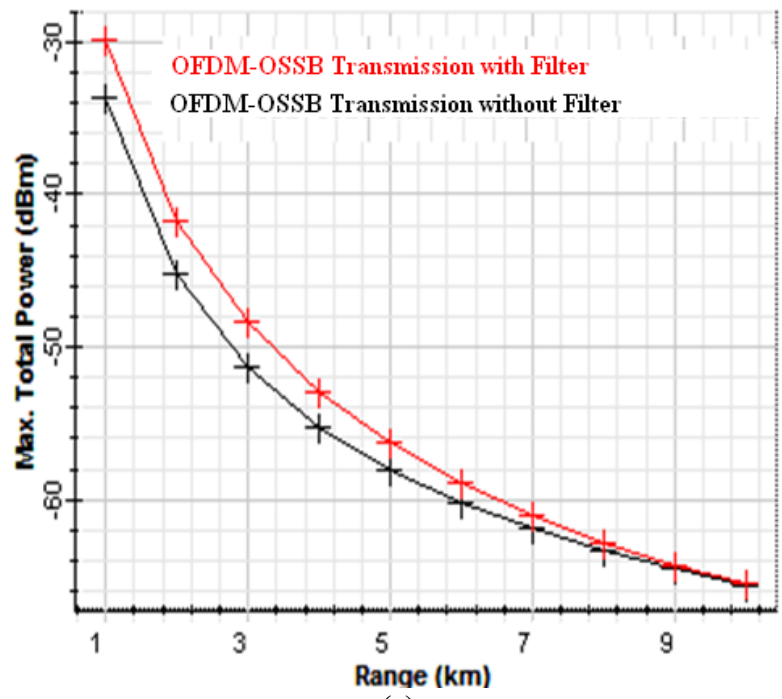

(c)

Fig 3: Evaluation of Total power vs. FSO link with (a) ODSB, (b) OTSB, and (c) OSSB modulation schemes

It is also observed that an improvement of $1.2 \mathrm{~dB}$ of $\mathrm{SNR}$ is achieved in case of OFDM-ODSB data transmission after transmitting 10Gbps data signals up to an FSO link of $3 \mathrm{~km}$ on comparing with OFDM-OSSB system as shown in Fig 1(c). But, beyond this, OFDM-OSSB system behaves better than the OFDM-OTSB system and shows an achievement of $0.33 \mathrm{~dB}$ improvement in SNR after an FSO link of $10 \mathrm{Km}$ and so, an improvement in total received power is achieved as shown in Fig 1 (d). Hence, it is clear from our results that OFDM-OSSB behaves well than that of other investigated modulation schemes in this work.

Further, an investigation of proposed FSO system using different modulation schemes with a Bessel filter connected after the MZM modulator is reported. It has been observed that an improvement in SNR of $4 \mathrm{~dB}$ in both the case of OFDM-ODSB- and OFDM-OTSB-system is achieved up to a FSO link of $4 \mathrm{Km}$ as shown in Fig 2(a, b). But, for OFDMOSSB system, an improvement of $2 \mathrm{~dB}$ is reported as shown in Fig 2(c). It has been also seen that after a FSO link of $10 \mathrm{Km}$, both the former systems shows the same behavior with a minute decrease in improvement of SNR. But, the impact of using Bessel filter in vanished in OFDM-OSSB system after a FSO link of $6 \mathrm{Km}$ as shown in Fig 2. The same behavior is also measured in case of total power received for all the cases of simulated modulation schemes as shown in Fig 3.

\section{CONCLUSION}

Our simulative results shows that the TSSB performs better than ODSB as the later one is to severely suffer with fading problems and OSSB performs in between the two under clear weather conditions. In case of using Bessel filter after MZM modulator along with ODSB, OSSB and OTSB schemes, a noticeable improvement in SNR and total power received is achieved with acceptable BER of $10^{-6}$ in this proposed integrated FSO system. So, it is recommended to design a FSO system using OFDM multicarrier technique along with OSSB modulation and a Bessel filter to achieve a prolonged FSO link at high data rate transmission.

\section{REFERENCES}

[1] Zin, A.M, Bongsu, M.S, Idrus. S.M, Zulkifl, N., "An overview of radio-over-fiber network technology", IEEE International Conference on Photonics (ICP), pp: 1-3, 2010.

[2] Chan, V.W.S., "Optical space communications: a key building block for wide area space networks", Lasers and Electro-Optics Society, volume 1, pp: 41-42, 1999.

[3] Chan, V.W.S., "Free-Space Optical Communications", Journal of Lightwave Technology, volume 24, Issue 12, pp: 4750-4762, 2006.

[4] Willebrand, H.A., Ghuman, B.S., "Fiber optics without fiber", Spectrum, IEEE, volume 38, Issue 8, pp: 40-45, 2001.

[5] R.K. Crane and Horng-Chung Shich, "A TwoComponent for the Prediction of Site Diversity Performance", Journal of Radio Science, volume 24, Issue 5, pp: 641-655, 1989.

[6] Zhuanhong Jia, Qinglin Zhu, Faliang Ao, "Atmospheric Attenuation Analysis in the FSO Link", International technology on Communication Technology (ICCT), pp: 1- 4, 2006.

[7] Hamed Al Raweshidy and Shozo Komaki, "Radio over fiber technologies for mobile communications networks", Artech House, London, 2002

[8] X. N. Fernando and S. Z. Pinter, "Radio over Fiber for Broadband Wireless Access", Department of Electrical and Computer Engineering, Ryerson University, Toronto, Canada, 2005.

[9] J.E. Mitchell, "Performance of OFDM at $5.8 \mathrm{GHz}$ using radio over fiber link," Electronics Letters, Vol. 40, No. 21, 2004

[10] Marwanto and S. M. Idrus, "SCM/WDM Radio over Fiber for Broadband Communication", INCOMTIS2008, Semarang Indonesia, 2008

[11] Bahai and B.R. Saltzberg, "Multi-carrier Digital Communication: Theory and Applications of OFDM". Norwell, MA: Kluwer, 1999.

[12] R. Prasad, "OFDM for Wireless Communications Systems," Artech House Publication, 2004.

[13] H. Sasa, T. Niiho, K. Tanaka, K. Utsumi and S. Morikura, "Radio-over-fiber transmission performance of OFDM signal for dual-band wireless LAN systems," MWP 2003, pp. 139-142, 2003.

[14] Kim, Y. Joo and Y. Kim, " $60 \mathrm{GHz}$ wireless communication systems with radio-over-fiber links for indoor wireless LANs," IEEE Trans. Consum. Electronics, vol. 50, no. 2, pp.517-520, 2004

[15] Chun-Ting Lin, Yu-Min Lin, Jason Chen, Sheng-Peng Dai, Peng-Chun Peng, Po Tsung, Shih Sien Chi, “ Generation of Direct-Detection Optical OFDM Signal for Radio-Over-Fiber Link using Frequency Doubling Scheme with Carrier Suppression" 2008

[16] Z. Jia, J. Yu, D. Qian, G. Ellinas, G.-K. Chang, "Experimental demonstration for delivering 1Gbps OFDM signals over 80-km SSMF in 40-GHz radio-overfiber access systems," OFC/NFOEC 2008, JWA108, 2008

[17] J. Yu, J. Hu, D. Qian, Z. Jia, G.-K. Chang, T. Wang, 16Gbps Super Broadband OFDM-Radio-over-Fiber System," OFC/NFOEC 2008, OThP2. 